\title{
Odderon with a running coupling constant
}

\author{
M.A.Braun \\ S.Peterburg State University, Russia
}

October 30, 2018

\begin{abstract}
The running coupling is introduced into the equation for the odderon via the bootstrap relation. It is shown that the previously found odderon state with a maximal intercept, which is constructed from antisymmetric pomeron wave function, continues to exist in the running coupling case. Its intercept is found to remain equal to unity independent of the behaviour assumed for the running coupling at low momenta.
\end{abstract}

\section{Introduction}

Lately a renewed interest has been shown towards introduction of a running coupling into the BFKL dynamics. Summation of contributions from quark- antiquark loops to the evolution of the gluon density has been used to restore the full dependence of the coupling on the running scale in the color dipole approach [1, 2, 3]. It turned out that the obtained kernel for the linear BFKL equation essentially coincides with the one which we found many years ago by imposing the bootstrap relation necessary for the fulfilment of unitarity [4, 5]. In this paper we draw attention to the fact that the bootstrap relation in fact allows to derive also the structure of the odderon in the perturbative QCD approach. It is of a particular interest to know what happens to the odderon state with a maximal intercept unity found in [6] and expressed via an antisymmetric pomeron state when a running coupling is introduced. We demonstrate that since the bootstrap relation plays a decisive role in construction of this solution to the odderon equation, introduction of the running coupling via the bootstrap allows to preserve this particular solution.

As in our earlier papers, we have to stress from the start that introduction of the running coupling into the BFKL formalism cannot be made rigorously and uniquely. The formalism admits transverse momenta of any magnitude, including very small ones, for which the concept of the gluon and its coupling looses any sense. The introduced running coupling has to be artificially continued to small momentum values, where it is completely undetermined. As a result the actual values for the pomeron and hence odderon intercepts generally depend on a chosen behaviour of the running coupling in the region of very low momenta. However particular odderon states constructed from antisymmetric pomeron states continue to have their maximal intercept exactly equal to unity, as in the fixed coupling case studied in [6]. 
Note that we use the bootstrap equation only in the lowest order of the coupling. So one can hope for only establishing the leading order behaviour in the running coupling. For the pomeron, in the next-to- leading order, it has been explicitly found that the bootstrap method correctly reproduces the part of the kernel responsible for the running of the coupling but not the rest piece [8, 9]. One expects the same to be true also for the odderon.

We limit ourselves to the case of the odderon at rest (with zero total momentum). Generalization to non-zero total momenta is straightforward.

\section{The running coupling from the bootstrap}

As mentioned in the Introduction, our idea to introduce a running coupling via the bootstrap was presented in [4, 5]. It consists in expressing both the gluon trajectory and intergluon interaction in terms of a single function $\eta(q)$ of the gluon momentum in the way which preserves the bootstrap relation. Function $\eta(q)$ then can be chosen to conform to the highmomentum behaviour of the gluon density with a running coupling. Let the two-gluon equation be written as an inhomogeneous Schroedinger equation

$$
(H-E) \psi=F
$$

with the Hamiltonian for the colour group $S U\left(N_{c}\right)$

$$
H=\frac{1}{2} N_{c}\left(t\left(q_{1}\right)+t\left(q_{2}\right)\right)+\left(T_{1} T_{2}\right) V .
$$

Here $\left(N_{c} / 2\right) t(q) \equiv-\omega(q)$ is the kinetic energy of the gluon given by its Regge trajectory with the minus sign. We write $t(q)$ in the form

$$
t(q)=\int \frac{d^{2} q_{1}}{(2 \pi)^{2}} \frac{\eta(q)}{\eta\left(q_{1}\right) \eta\left(q-q_{1}\right)},
$$

where $\eta(q)$ is an arbitrary function satisfying the condition

$$
\eta(0)=0,
$$

which guarantees that the gluon trajectory passes through zero at $q=0$ in accordance with the gluon properties. The interaction term, apart from the product of the gluon colour vectors $T_{i}^{a}, i=1,2, a=1, \ldots N_{c}$, involves the interaction kernel $V$ which we also express via the same function $\eta(q)$

$$
V\left(q_{1}, q_{2} \mid q_{1}^{\prime} q_{2}^{\prime}\right)=\frac{\eta\left(q_{1}+q_{2}\right)}{\eta\left(q_{1}^{\prime}\right) \eta\left(q_{2}^{\prime}\right)}-\left(\frac{\eta\left(q_{1}\right)}{\eta\left(q_{1}^{\prime}\right)}+\frac{\eta\left(q_{2}\right)}{\eta\left(q_{2}^{\prime}\right)}\right) \frac{1}{\eta\left(q_{1}-q_{1}^{\prime}\right)} .
$$

It conserves the momentum: $q_{1}+q_{2}=q_{1}^{\prime}+q_{2}^{\prime}$. Comparing (3) with (15) one finds the bootstrap relation, satisfied for arbitrary $\eta(q)$ :

$$
\int \frac{d^{2} q_{1}}{(2 \pi)^{2}} V\left(q_{1}, q_{2} \mid q_{1}^{\prime}, q_{2}^{\prime}\right)=t\left(q_{1}\right)+t\left(q_{2}\right)-t\left(q_{1}+q_{2}\right) .
$$


From the high-momentum behaviour of the gluon distribution with a running coupling one finds

$$
\eta(q)=\frac{1}{2 \pi} b q^{2} \ln \frac{q^{2}}{\Lambda^{2}}, \quad q^{2}>>\Lambda^{2},
$$

where $\Lambda$ is the standard QCD parameter and

$$
b=\frac{1}{12}\left(11 N_{c}-2 N_{f}\right) .
$$

The asymptotic (7) and condition (4) are the only properties of $\eta(q)$ which follow from the theoretical reasoning. A concrete form of $\eta(q)$ interpolating between (44) and (7) may be chosen differently. One hopes that physical results will not too strongly depend on the choice.

The fixed coupling case corresponds to the choice

$$
\eta(q)=\frac{2 \pi}{g^{2}} q^{2} .
$$

Then one finds the standard BFKL expressions for the trajectory $t(q)$ and interaction $V\left(q_{1}, q_{2} \mid q_{1}^{\prime}, q_{2}^{\prime}\right)$.

It is customary to discuss a homogeneous equation (11) (with $F=0$ ) to seek for the ground state energy, which determines the rightmost singularity in the complex angular momentum $j$ related to the energy by

$$
j=1-E
$$

For our purpose it is, however, essential to conserve the inhomogeneous term $F\left(q_{1}, q_{2}\right)$, which represents the two-gluon-particle vertex.

Consider the vector colour channel with $T_{1} T_{2}=-N_{c} / 2$ and, most important, assume that the inhomogeneous term depends only on the total momentum of the two gluons: $F=F\left(q_{1}+q_{2}\right)$. Then using the bootstrap relation (6) one easily finds the solution to Eq. (1):

$$
\psi\left(q_{1}, q_{2}\right)=\psi\left(q_{1}+q_{2}\right)=\frac{F\left(q_{1}+q_{2}\right)}{\omega\left(q_{1}+q_{2}\right)-E} .
$$

It means that the two gluons 1 and 2 have fused into a single one with the momentum $q_{1}+q_{2}$. This phenomenon is true for aribitrary $\eta(q)$.

\section{The odderon}

\subsection{The bootstrap for three gluons}

Noe we generalize the bootstrap for two gluons to the case of three gluons, relevant for the odderon. For three gluons Eq. (1) holds with the Hamiltonian which is a sum of kinetic energies and pair interactions:

$$
H=\frac{1}{2} N_{c} \sum_{i=1}^{3} t\left(q_{i}\right)+\sum_{i<k}^{3}\left(T_{i} T_{k}\right) V_{i k} .
$$


Here $V_{i k}$ is the interaction of gluons $i$ and $k$ with the kernel $V\left(q_{i}, q_{k} \mid q_{i}^{\prime}, q_{k}^{\prime}\right)$. We do not impose any restrictions on the total colour $T=\sum_{i=1}^{3} T_{i}$. (However only for $T=0$ the Hamiltonian is infrared stable).

Assume now that $T_{1} T_{2}=-N_{c} / 2$, i.e. the gluons 1 and 2 form a colour vector. Then we demonstrate that for a certain specific choice of the inhomogeneous term $F$ Eq. (11) for 3 gluons reduces to that for two gluons, the gluons 1 and 2 fused into a single gluon which carries their total momentum and colour. In other words, as in the two-gluon case, a pair of gluons in the adjoint representation is equivalent to a single gluon.

Of course, the specific form of the inhomogeneous term is a decisive instrument for bootstrapping the two gluons 1 and 2. For three gluons we choose

$$
F_{3}\left(q_{1}, q_{2}, q_{3}\right)=\int \frac{d^{2} q_{3}^{\prime}}{(2 \pi)^{2}} \hat{W}\left(q_{1}, q_{2}, q_{3} \mid q_{1}^{\prime}, q_{3}^{\prime}\right) \psi_{2}\left(q_{1}^{\prime}, q_{3}^{\prime}\right)+F_{2}\left(q_{1}+q_{2}, q_{3}\right)
$$

with $q_{1}^{\prime}+q_{3}^{\prime}=q_{1}+q_{2}+q_{3}$. In Eq. (13) $\psi_{2}$ is a solution of the Schroedinger equation (1) with the inhomogeneous term $F_{2}$. Gluon 1 in it substitutes the fused initial gluons 1 and 2 . The kernel $\hat{W}$ is an operator acting also on colour indeces of $\psi_{2}$. It is convenient to retain a pair of colour indeces for gluon 1 in $\psi_{2}$ inherited from the initial gluons 1 and 2 by means of a projector onto the adjoint representation in the colour $T_{1}+T_{2}$. This allows to consider $\hat{W}$ as an operator acting in the colour space of three gluons (not two). Then it has the form

$$
\hat{W}\left(q_{1}, q_{2}, q_{3} \mid q_{1}^{\prime}, q_{i}^{\prime}\right)=\left(T_{1} T_{3}\right) W\left(q_{2}, q_{1}, q_{3} \mid q_{1}^{\prime}, q_{3}^{\prime}\right)+\left(T_{2} T_{3}\right) W\left(q_{1}, q_{2}, q_{3} \mid q_{1}^{\prime}, q_{3}^{\prime}\right),
$$

where the momentum space kernel $W$ is a difference between two kernels (5), expressed via function $\eta(q)$ :

$$
W\left(q_{2}, q_{1}, q_{3} \mid q_{1}^{\prime}, q_{3}^{\prime}\right)=V\left(q_{1}, q_{3} \mid q_{1}^{\prime}-q_{2}, q_{3}^{\prime}\right)-V\left(q_{1}+q_{2}, q_{3} \mid q_{1}^{\prime}, q_{i}^{\prime}\right) .
$$

The explicit form of $W$ is irrelevant for our purpose. In terms of function $\eta(q)$ it can be found in [10]. It possesses a symmetry property, which is important for us:

$$
W\left(q_{1}, q_{2}, q_{3} \mid q_{1}^{\prime}, q_{3}^{\prime}\right)=W\left(q_{3}, q_{2}, q_{1} \mid q_{3}^{\prime}, q_{1}^{\prime}\right) \text {. }
$$

Note that in the fixed coupling case this kernel reduces to the Bartels kernel $K_{2 \rightarrow 3}$ which describes transition from two BFKL pomerons to three [11.

We are going to show that, with the inhomogeneous term $F_{3}$ given by Eq. (13) and $T_{1} T_{2}=-N_{c} / 2$, the Schroedinger equation (1) for three gluons is solved by

$$
\psi_{3}\left(q_{1}, q_{2}, q_{3}\right)=\psi_{2}\left(q_{1}+q_{2}, q_{3}\right) .
$$

Indeed putting (17) into the equation we find that the interaction term $\left(T_{1} T_{2}\right) V_{12}$, according to (6), substitutes the sum of kinetic terms $t\left(q_{1}\right)+t\left(q_{2}\right)$ for the gluons 1 and 2 by $t\left(q_{1}+q_{2}\right)$, which is precisely the kinetic term for the function $\psi_{2}\left(q_{1}+q_{2}\right)$. The interaction of gluon 1 with the third one takes the form

$$
\left(T_{1} T_{3}\right) \int \frac{d^{2} q_{3}^{\prime}}{(2 \pi)^{2}} V\left(q_{1}, q_{3} \mid q_{1}^{\prime}, q_{3}^{\prime}\right) \psi_{2}\left(q_{1}^{\prime}+q_{2}, q_{3}^{\prime}\right)=
$$




$$
\left(T_{1} T_{3}\right) \int \frac{d^{2} q_{3}^{\prime}}{(2 \pi)^{2}} V\left(q_{1}, q_{3} \mid q_{1}^{\prime}-q_{2}, q_{3}^{\prime}\right) \psi_{2}\left(q_{1}^{\prime}, q_{3}^{\prime}\right) .
$$

The momentum is conserved during the interaction, so that on the lefthand side $q_{1}+q_{3}=$ $q_{1}^{\prime}+q_{3}^{\prime}$ and on the righthand side $q_{1}+q_{2}+q_{3}=q_{1}^{\prime}+q_{3}^{\prime}$. The term (18) is cancelled by the identical contribution with the opposite sign coming from the inhomogeneous term $F_{3}$ (the first term in (15) in the part of $\hat{W}$ proportional to $T_{1} T_{3}$ ). Instead of it from the inhomogeneous term comes the contribution (the second term in (15))

$$
\left(T_{1} T_{3}\right) \int \frac{d^{2} q_{3}^{\prime}}{(2 \pi)^{2}} V\left(q_{1}+q_{2}, q_{3} \mid q_{1}^{\prime}, q_{3}^{\prime}\right) \psi_{2}\left(q_{1}^{\prime}, q_{3}^{\prime}\right) .
$$

In the same manner the interaction of gluon 2 with the third one

$$
\begin{gathered}
\left(T_{2} T_{3}\right) \int \frac{d^{2} q_{3}^{\prime}}{(2 \pi)^{2}} V\left(q_{2}, q_{3} \mid q_{2}^{\prime}, q_{3}^{\prime}\right) \psi_{2}\left(q_{1}+q_{2}^{\prime}, q_{3}^{\prime}\right)= \\
\left(T_{2} T_{i}\right) \int \frac{d^{2} q_{3}^{\prime}}{(2 \pi)^{2}} V\left(q_{2}, q_{3}, q_{2}^{\prime}-q_{1}, q_{3}^{\prime}\right) \psi_{2}\left(q_{2}^{\prime}, q_{3}^{\prime}\right)
\end{gathered}
$$

is transformed by the term in $F_{3}$ proportional to $T_{2} T_{3}$ into

$$
\left(T_{2} T_{3}\right) \int \frac{d^{2} q_{3}^{\prime}}{(2 \pi)^{2}} V\left(q_{1}+q_{2}, q_{3} \mid q_{1}^{\prime}, q_{3}^{\prime}\right) \psi_{2}\left(q_{1}^{\prime}, q_{3}^{\prime}\right) .
$$

The two terms (18) and (21) sum into

$$
\left(T_{1}+T_{2}, T_{3}\right) \int \frac{d^{2} q_{3}^{\prime}}{(2 \pi)^{2}} V\left(q_{1}+q_{2}, q_{3} \mid q_{1}^{\prime}, q_{3}^{\prime}\right) \psi_{2}\left(q_{1}^{\prime}, q_{3}^{\prime}\right),
$$

which is precisely the correct form for the interaction of the gluon with the total colour $T_{1}+T_{2}$ and momentum $q_{1}+q_{2}$ in which the initial gluons 1 and 2 have fused. As a result we obtain the Schroedinger equation (1) for the function $\psi_{2}$ describing two gluons with the inhomogeneous term $F_{2}$.

The inhomogeneous term of the form (13) actually appears in the theory of three reggeized gluons, provided one takes into account the possibility for transitions from 2 to 3 gluons. In the case of the fixed coupling constant this fact was discovered in the course of the perturbative analysis of the system of 4 reggeized gluons in the triple Regge kinematical region [12, 13]. Introducing a running coupling via the bootstrap allows to preserve this result for a general choice of function $\eta(q)$.

We stress that this result is valid for any value of the total colour of the three-gluon system and any choice of the symmetry of the spatial wave function. In the particular case of the colorless three-gluon system with a $d$-structure of the color wave function (the odderon state) the bootstrap allows to find a specific solution to the odderon equation, expressed via an antisymmetric pomeron state. 


\section{$3.2 \quad$ A solution of the odderon equation}

In the case of the odderon the total colour is zero, so that any pair of gluons is in the colour vector state and $T_{i} T_{k}=-N_{c} / 2$ for $i<k=1,2,3$. The total color wave function is $d_{a_{1} a_{2} a_{3}}$ where $a_{i}$ is the color of the $i$-th reggeized gluon. Separating this color factor one finds the equation for the odderon momentum wave function

$$
\left(H_{o d}-E\right) \psi_{o d}=0,
$$

with a Hamiltonian

$$
H^{o d}=\frac{1}{2} N_{c}\left(\sum_{i=1}^{3} t\left(q_{i}\right)-\sum_{i<k}^{3} V_{i k}\right) .
$$

Now we use the bootstrap results obtained above. Consider the odderon equation with the inhomogeneous term (13) in which $T_{1} T_{3}=T_{1} T_{2}=-N_{c} / 2$, the colour wave function $d_{a_{1} a_{2} a_{3}}$ and $F_{2}=0$. Our result tells us that this equation is solved by the pomeron momentum wave function

$$
\psi^{(12)}=\psi_{2}\left(q_{1}+q_{2}, q_{3}\right)
$$

which satisfies the homogeneous Schroedinger equation (1) with a Hamiltonian (2) in which $T_{1} T_{2}=-N_{c}$ (the BFKL equation in the case of a fixed coupling). Explicitly we find that in the momentum space

$$
\left(H_{o d}-E\right) \psi^{(12)}=F_{3}^{(12)},
$$

where

$$
F_{3}^{(12)}(1,2,3)=-\frac{1}{2} N_{c} \int \frac{d^{2} q_{3}^{\prime}}{(2 \pi)^{2}}\left(W\left(2,1,3 \mid 1^{\prime}, 3^{\prime}\right)+W\left(1,2,3 \mid 1^{\prime}, 3^{\prime}\right)\right) \psi_{2}\left(1^{\prime}, 3^{\prime}\right) .
$$

Here and in the following for brevity we denote gluon momenta just by numbers: $1 \equiv q_{1}$, $1^{\prime} \equiv q_{1}^{\prime}$ etc.

Next we cyclically permute the gluons $1,2,3$ to obtain two more relations

$$
\left(H_{o d}-E\right) \psi^{(23)}=F_{3}^{(23)}
$$

and

$$
\left(H_{o d}-E\right) \psi^{(31)}=F_{3}^{(31)},
$$

where for instance

$$
\psi^{(23)}=\psi_{2}\left(q_{2}+q_{3}, q_{1}\right)
$$

and

$$
F_{3}^{(23)}(1,2,3)=-\frac{1}{2} N_{c} \int \frac{d^{2} q_{3}^{\prime}}{(2 \pi)^{2}}\left(W\left(3,2,1 \mid 1^{\prime}, 3^{\prime}\right)+W\left(2,3,1 \mid 1^{\prime}, 3^{\prime}\right)\right) \psi_{2}\left(1^{\prime}, 3^{\prime}\right)
$$

(the integration momenta $q_{3}^{\prime}$ and $q_{1}^{\prime}=q_{1}+q_{2}+q_{3}-q_{3}^{\prime}$ do not change under permutations of the external momenta). We add all the three Schroedinger equations obtained in this manner together to obtain

$$
\left(H_{o d}-E\right)\left(\psi^{(12)}+\psi^{(23}+\psi^{(31)}\right)=F_{3}^{(12)}+F_{3}^{(23)}+F_{3}^{(31)} \equiv F_{3}^{\text {tot }} .
$$


The total inhomogeneous term has a structure

$$
F_{3}^{\text {tot }}(1,2,3)=-\frac{N_{c}}{2} \int \frac{d^{2} q_{3}^{\prime}}{(2 \pi)^{2}} U\left(1,2,3 \mid 1^{\prime}, 3^{\prime}\right) \psi_{2},\left(1^{\prime}, 3^{\prime}\right)
$$

where

$$
\begin{gathered}
U\left(1,2,3 \mid 1^{\prime}, 3^{\prime}\right)=W\left(2,1,3 \mid 1^{\prime}, 3^{\prime}\right)+W\left(1,2,3 \mid 1^{\prime}, 3^{\prime}\right)+W\left(3,2,1 \mid 1^{\prime}, 3^{\prime}\right) \\
+W\left(2,3,1 \mid 1^{\prime}, 3^{\prime}\right)+W\left(1,3,2 \mid 1^{\prime}, 3^{\prime}\right)+W\left(3,1,2 \mid 1^{\prime}, 3^{\prime}\right) .
\end{gathered}
$$

Using property (16) one easily finds that $U$ is symmetric in the last pair of arguments

$$
U\left(1,2,3 \mid 1^{\prime}, 3^{\prime}\right)=U\left(1,2,3 \mid 3^{\prime}, 1^{\prime}\right) .
$$

It follows that if function $\psi_{2}\left(q_{1}, q_{2}\right)$ is antisymmetric in its variables, the total inhomogeneous term (33) in Eq. (32) vanishes and the sum

$$
\psi_{\text {od }}=\psi^{(12)}+\psi^{(23}+\psi^{(31)}
$$

is a solution of the odderon equation

$$
\left(H_{o d}-E\right) \psi_{o d}=0
$$

Thus from any antisymmetric soloution of the pomeron equation one gets a corresponding solution of the odderon equation.

For a fixed coupling this result was found in [6]. Our derivation generalizes it to the case of a running coupling, provided it is introduced in the manner which preserves the bootstrap relations.

\section{The pomeron and odderon intercepts}

With the running coupling constant the intercept of the pomeron states generally begins to depend on the way in which the constant is chosen to behave at small values of momenta (where in fact it is not defined physically). We recall that our function $\eta(q)$ is fixed only by its asymptotic behaviour at large values of $q$ (17) and its value at $q=0$ (44). A possible simple form of $\eta(q)$ satisfying these conditions and no more singular at $q=0$ than in the fixed coupling case is

$$
\eta(q)=\frac{1}{2 \pi} b q^{2} \ln \left(\frac{q^{2}}{\Lambda^{2}}+\xi^{2}\right)
$$

with two parameters, the standard QCD parameter $\Lambda$ and $\xi>1$, constrained by the experimental value of the coupling constant $\alpha_{s}\left(q^{2}\right)$ at some chosen $q^{2}$ value. This form implies that the running coupling $\alpha_{s}(q)$ steadily grows towards small values of $q$ and freezes at $q \sim q_{0} \sim \xi \Lambda$ to have its value at $q=0$

$$
\alpha_{s}(0)=\frac{\pi}{2 b \ln \xi} .
$$


Choosing $m_{w}$ as the experimental scale and taking $\alpha_{s}\left(m_{w}^{2}\right)=0.126$ (to have $\Lambda \simeq 0.2 \mathrm{GeV} / \mathrm{c}$ at small values of $\xi$, in the one-loop approximation) we find that the interval of possible values of $\xi$ is $1 \leq \xi \leq \xi_{\max }=1.57 \cdot 10^{5}$. As $\xi$ rises, so do $\Lambda$ and $q_{0}$, whereas $\alpha_{s}(0)$ and the intercept $\Delta$ steadily diminish towards their limiting values at $\xi_{\max }, 0.126$ and 0.330 respectively. Values of all these quantities at different $\xi$ are presented in the Table.

Table. Pomeron intercepts

\begin{tabular}{|r|r|r|r|r|}
\hline$\xi$ & $\Lambda(\mathrm{GeV} / \mathrm{c})$ & $\alpha_{s}(0)$ & $q_{0}(\mathrm{GeV} / \mathrm{c})$ & $\Delta$ \\
\hline 2.0 & 0.197 & 2.18 & 0.278 & 5.69 \\
$2.0 \cdot 10^{2}$ & 0.197 & 0.284 & 2.78 & 0.743 \\
$2.0 \cdot 10^{3}$ & 0.198 & 0.198 & 8.84 & 0.516 \\
$2.0 \cdot 10^{4}$ & 0.210 & 0.152 & 29.7 & 0.395 \\
$5.0 \cdot 10^{4}$ & 0.237 & 0.139 & 53.2 & 0.361 \\
$9.0 \cdot 10^{4}$ & 0.300 & 0.132 & 90.0 & 0.343 \\
$1.5 \cdot 10^{5}$ & 0.893 & 0.127 & 346 & 0.330 \\
\hline
\end{tabular}

In this respect it is worth noting that introduction of the running coupling does not eliminate the arbitrariness in the value chosen for the coupling in the fixed coupling case, just trading this arbitrariness for the one in the choice of, say, $\alpha_{s}(0)$.

However it is remarkable that introduction of the running coupling does not change the maximal intercept of the odderon, which, with unity subtracted, remains equal to zero. In the fixed coupling case the maximal intercept of antisymmetric pomeron states is unity $(E=0)$. Correspondingly the odderon built from these states according to Eq. (36) has its maximal intercept equal to unity. It is trivial to show that introduction of a running coupling does not change this result.

Indeed consider the Schroedinger equation (11) for the forward pomeron: $q_{1}+q_{2}=0$, $T_{1} T_{2}=-N_{c}$. Divided by $N_{c}$, in terms of function $\eta(q)$ this equation for the semi-amputated function

$$
\phi(q)=\eta(q) \psi_{2}(q)
$$

has the form

$$
\phi(q) \int \frac{d^{2} q_{1}}{(2 \pi)^{2}} \frac{\eta(q)}{\eta\left(q_{1}\right) \eta\left(q-q_{1}\right)}-2 \int \frac{d^{2} q_{1}}{(2 \pi)^{2}} \frac{1}{\eta\left(q-q_{1}\right)} \phi\left(q_{1}\right)=E \phi(q) .
$$

This equation has an obvious antisymmetric solution

$$
\phi(q)=\frac{\mathbf{q}}{\eta(q)}
$$

corresponding to the eigenvalue $E=0$. Indeed with (42) the left-hand side of (41) acquires the form

$$
\mathbf{q} \int \frac{d^{2} q_{1}}{(2 \pi)^{2}} \frac{1}{\eta\left(q_{1}\right) \eta\left(q-q_{1}\right)}-2 \int \frac{d^{2} q_{1}}{(2 \pi)^{2}} \frac{\mathbf{q}_{1}}{\eta\left(q_{1}\right) \eta\left(q-q_{1}\right)} .
$$


In the second term, the change of variables $q_{1} \rightarrow q-q_{1}$ transforms the numerator into $\mathbf{q}-\mathbf{q}_{\mathbf{1}}$. The half of the sum of these equal expressions coincides with the first term in (43) so that the left-hand side vanishes. Thus (42) is indeed a solution of Eq. (41) with a zero eigenvalue.

\section{Conclusions}

Introduction of the running coupling via the bootstrap relation advocated for the pomeron a long time ago in 4, 5] allows to construct in a straightforward manner also an equation for the odderon with a running coupling. Its form admits solutions expressed via antisymmetric pomeron states, similar to the ones found in the fixed coupling case. The maximal value of the intercept for such solutions remains equal to unity as in the fixed coupling case.

\section{Acknowledgements}

This work has been supported by grants RNP 2.1.1.1112 and RFFI 06-02-16115a of Russia.

\section{References}

[1] Yu.V.Kovchegov and H.Weigert, hep-ph/0609090.

[2] Yu.V.Kovchegov and H.Weigert, hep-ph/0612071.

[3] I.I.Balitsky, hep-ph/0609087.

[4] M.A.Braun, Phys.Lett. B 345 (1995) 155.

[5] M.A.Braun, Phys.Lett. B 348 (1995) 190.

[6] J.Bartels, L.N.Lipatov and G.P.Vacca, Phys. Lett. B 477 (2000) 178.

[7] J.Bartels, Nucl. Phys, B 175 (1980) 365.

[8] M.A.Braun and G.P.Vacca, Phys. Lett. bf B 454 (1999) 319; B 447 (2000) 156.

[9] V.S.Fadin, R.Fiore and A.Papa, Phys. Rev. D 60 (1999) 074025.

[10] M.A.Braun, hep-ph/0703006 (to be published in Eur. Phys. J C).

[11] J.Bartels, Nucl.Phys. B 175 (1980) 365.

[12] J.Bartels, Z.Phys. C 60 (1993) 471.

[13] J.Bartels and M.Wuesthoff, Z.Phys. C 66 (1995) 157. 